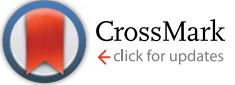

Cite this: RSC Adv., 2017, 7, 5488

Received 28th October 2016

Accepted 5th January 2017

DOI: 10.1039/c6ra25986c

www.rsc.org/advances

\title{
Lignin-assisted double acoustic irradiation for concentrated aqueous dispersions of carbon nanotubes
}

\begin{abstract}
Sheila M. Goodman, $\uparrow$ Noah Ferguson $\uparrow$ and Anthony B. Dichiara*
The full potential of carbon nanotubes (CNTs), one of the most widely used nanomaterials to date, still remains to be realized, and the dispersion of CNTs is one of the main challenging tasks for many practical applications. Lignin, one of the most abundant renewable polymers, has recently been investigated as a potential dispersant to prepare CNT suspensions. The present study provides a benchmark of the effectiveness of lignin in dispersing CNTs compared to typical petrochemical surfactants. Aqueous dispersions of CNTs are characterized by UV-vis spectroscopy, optical, and electronic microscopy. The influence of dispersant concentration, sonication energy, surfactant mixtures, CNT morphology and oxidation are thoroughly examined. The use of a double acoustic irradiation system combining both bath and probe sonication is also investigated as a promising way to improve dispersion quality and reduce processing time. Results demonstrate that lignin-assisted double sonication represents a major low-cost and renewable alternative to prepare stable and concentrated suspensions of individualized CNTs.
\end{abstract}

\section{Introduction}

Achieving concentrated aqueous dispersions of carbon nanotubes (CNTs) is critical for practical applications, such as polymer composites, ${ }^{1}$ ceramic materials, ${ }^{2}$ and coatings. ${ }^{3}$ However, the hydrophobicity of CNTs and their tendency to readily form bundles by hexagonal packing of individual nanotubes along their length axis with high van der Waals binding energy, significantly hampers the properties of CNT-based products. ${ }^{4,5}$ Hence, the individualization of CNT aggregates is necessary to take full advantage of the potential of CNTs. For instance, uniform dispersions of CNTs are desired to provide larger numbers of charge transport routes in polymer composites, such that a much lower percolation threshold for electrical conductance can be achieved. ${ }^{6-8}$ Various techniques to disperse and debundle CNTs have been developed, including high shear mixing, sonication, and ultracentrifugation. ${ }^{9}$ These methods can be divided into two different categories depending on the nature of the CNT surface modification used to improve the dispersion of CNTs. On one hand, covalent functionalization aims at disrupting the stacking properties of CNTs by grafting functional groups on the side walls of CNTs to alter the hydrogen bonding and intercalate chemical species between the bundles..$^{10}$ On the other hand, noncovalent functionalization involves the physical adsorption of surfactants, polymers,

School of Environmental and Forest Sciences, University of Washington, Seattle, WA 98195, USA. E-mail: abdichia@uw.edu; Tel: +1 2065431581

$\dagger$ S. Goodman and N. Ferguson equally contributed to this work.
DNA, and peptides. ${ }^{11-13}$ The noncovalent bonding of surfactants with CNTs is typically considered as a superior approach since it does not modify the $\pi$-electron network of CNTs and preserves their structural integrity. In addition, surfactants can be relatively easily removed after treatment by washing. A large variety of surfactants, such as sodium dodecyl-benzenesulphonate (SDBS),${ }^{14}$ octyl phenol ethoxylate (Triton X-100), ${ }^{15}$ cetyltrimethylammonium bromide (CTAB) ${ }^{16}$ and sodium dodecyl sulfate (SDS) ${ }^{17}$ have been examined for the dispersion of CNTs in aqueous solutions under the noncovalent approach. However, the great majority of surfactants are petroleum-based chemicals. As environmental concerns rise and oil prices continue to trend upward due to diminishing supply, a greater emphasis on renewable resources is needed to explore new classes of dispersants to prepare CNT solutions.

Lignin is the second most abundant natural polymer and represents up to $30 \%$ of the world organic biomass. Despite its widespread availability, the applications of lignin are rather limited. Currently, lignin is an underutilized byproduct of the pulp and paper industry and often treated as a waste that is burned for energy recovery. Lignin was discovered in 1838 by Anselme Payen and consists of a three-dimensional, highly cross-linked, phenylpropane-type macromolecule mainly comprised of carbon and oxygen with no structural regularity within its polymeric framework. ${ }^{18}$ Due to its amphiphilic nature and the possibility of $\pi-\pi$ interactions with graphitic structures, opportunities exist to use lignin as a renewable dispersing agent for the preparation of aqueous solutions of CNTs. While lignin was recently found to promote the debundling of CNTs in 
water, ${ }^{19,20}$ no comparative assessment of the dispersion quality achieved using lignin and other typical petroleum-based surfactants under identical experimental conditions has been reported to date.

The present research provides a benchmark of the effectiveness of lignin in dispersing CNTs. Aqueous suspensions of multiwalled CNTs are examined using alkali lignin (AL), SDS and CTAB. UV-vis spectroscopy, optical, and electronic microscopy are employed to assess the dispersion state and stability of CNTs in water. The influence of sonication energy, dispersant concentration, CNT morphology and oxidation are analyzed. Acoustic irradiation using an innovative system combining both bath and probe sonication is also studied. Finally, binary and ternary mixtures of different mixing ratios of $\mathrm{AL} / \mathrm{SDS}, \mathrm{AL} / \mathrm{CTAB}$, and $\mathrm{AL} / \mathrm{SDS} / \mathrm{CTAB}$ are prepared to investigate possible synergistic effects that can improve surfactant adsorption on CNT surfaces and modifications of repulsive/attractive forces. This research provides relevant new insights to optimize CNT dispersions using renewable bio-based compounds.

\section{Experimental details}

\section{Materials}

Pristine and hydroxyl-functionalized CNTs with length of 10-20 $\mu \mathrm{m}$ and outer diameters below $8 \mathrm{~nm}$ and above $50 \mathrm{~nm}$ synthesized by catalytic chemical vapor deposition and purified using acid chemistry were purchased from Cheap Tubes Inc. The hydroxyl-functionalized CNTs per the manufacturer technical data contain $5.5 \%$ of $\mathrm{OH}$ groups. The main characteristics of the CNTs used in this study, including length, inner and outer diameter, and specific surface area are summarized in Table 1. All CNTs were used as received without any further treatment. The alkali lignin (AL, 99\%), sodium dodecyl sulfate (SDS, 99\%) and cetyltrimethylammonium bromide (CTAB, 99\%) dispersants were obtained from Tokyo Chemical Industry Co., MP Biomedicals, and Amresco, respectively. All dispersions were conducted using de-ionized (DI) water.

\section{Method}

A desired amount of dispersant was added to DI water to compare the dispersive efficiency of AL, SDS, and CTAB. Once the dispersant was completely dissolved after 10 minutes of bath sonication at room temperature $\left(25^{\circ} \mathrm{C}\right)$, CNTs were added into the solutions at a constant concentration of $1 \mathrm{wt} \%$ (CNT powder weight per water weight), which is more concentrated than typical suspensions of CNTs reported in the literature. ${ }^{21}$ The solution $\mathrm{pH}$ was kept at the natural level in all experiments (i.e. $\mathrm{pH} \sim 7$ ). CNTs with different outer diameters (8 and $50 \mathrm{~nm}$ ) and functional groups (pristine and $\mathrm{OH}$-functionalized) were examined. For mixed dispersant systems, binary and ternary mixtures of different mixing ratios of $\mathrm{AL} / \mathrm{SDS}, \mathrm{AL} / \mathrm{CTAB}$, and $\mathrm{AL} /$ SDS/CTAB were prepared under similar conditions. Unless otherwise specified, all CNTs dispersions were sonicated in an ice bath for 30 minutes using a $750 \mathrm{~W}$ Sonics Vibracell VCX probe sonicator equipped with a $13 \mathrm{~mm}$ stepped tip and running at $20 \%$ of maximum amplitude. The as-sonicated samples were then centrifuged at $5000 \mathrm{rpm}$ for 30 minutes to remove large undispersed clusters. The supernatants were diluted appropriately and probe sonicated for an additional 15 minutes at $20 \%$ amplitude for further comparative analysis by UV-vis absorption spectroscopy. To study the colloidal stability of the different CNT dispersions, the samples were stored at room temperature, in the dark, without agitation, and periodical measurements were taken over a 34 day time period.

\section{Characterization}

UV-vis measurements were performed in quartz cuvettes at room temperature $\left(25^{\circ} \mathrm{C}\right)$ using a Perkin Elmer Lambda 750 spectrophotometer operating in the $300-1200 \mathrm{~nm}$ range. The blanks were obtained using the original dispersant solutions diluted by the same factor and under the same conditions as the samples themselves. Indirect optical observations of the CNT dispersions were achieved by drop casting the different suspensions onto glass slides for observation using a Zeiss Axiocam ERc5s digital camera mounted on a Zeiss Axiolab light microscope. Direct imaging was conducted using a wet transmission electron microscopy (TEM) technique to assess the dispersion state of CNTs in suspensions. Unlike conventional TEM, the wet TEM method can overcome the inherent limitations induced by drying and exposing the sample to vacuum before imaging, hence allowing for representative in situ images of the dispersions to be taken without altering the original conditions of the fluid. The wet-cell was constructed by holding the solution between two silicon nitride membrane window TEM grids, and observed using a FEI TECNAI G2 F20 S-TWIN high resolution TEM with a beam acceleration voltage of 200 $\mathrm{keV}$.

\section{Results and discussion}

\section{Influence of dispersant concentration}

UV-vis spectroscopy is one of the most commonly used techniques to evaluate the quality of CNT dispersions. ${ }^{22}$ It involves the quantification of the portion of light absorbed as it travels

Table 1 Length, inner/outer diameter, and surface area of the various CNTs used in this work

\begin{tabular}{|c|c|c|c|c|}
\hline Sample & Length $(\mu \mathrm{m})$ & Inner diameter (nm) & Outer diameter (nm) & Specific surface area $\left(\mathrm{m}^{2} \mathrm{~g}^{-1}\right)$ \\
\hline 8-CNT & $10-20$ & $2-5$ & $<8$ & 500 \\
\hline $50-\mathrm{CNT}$ & $10-20$ & $5-10$ & $50-80$ & 60 \\
\hline 50-CNT-OH & $10-20$ & $5-10$ & $50-80$ & 60 \\
\hline
\end{tabular}


through the CNT suspension, relative to its blank counterpart. Individual disentangled CNTs are active in the UV-vis region and display characteristic bands corresponding to absorption due to 1D Van Hove singularities. ${ }^{23}$ However, the photoluminescence that is normally detected by UV-vis spectroscopy is quenched in bundled CNTs, meaning that CNT aggregates hardly absorb light in the wavelength range between 300 and $1200 \mathrm{~nm} .{ }^{24}$ Based on this principle, the degree of individualization of CNTs in aqueous solution can be related to the intensity of the corresponding absorption spectrum. ${ }^{25}$ For comparison purposes, the UV-vis spectra of aqueous CNT dispersions using different AL, SDS and CTAB concentrations are recorded, as illustrated in Fig. 1. Representative photographs of sonicated CNT aqueous suspensions with the different dispersants are shown in the insets (Fig. 1a-c) and reveal the Tyndall effect, which is indicative of colloidal systems. ${ }^{26}$ In each case, the absorbance in the UV-vis region rises with the addition of dispersant as compared to CNT suspensions prepared without any surfactant. In particular, it can be observed that the absorbance intensity increases with the dispersant concentration up to a certain level beyond which a further increase in concentration leads to a reduction of the absorbance intensity. The optimal dispersant concentrations yielding the highest absorbance under the considered conditions are determined to be $2 \mathrm{wt} \%$ for both $\mathrm{AL}$, SDS and CTAB, which is higher than their critical micelle concentrations $(6 \mathrm{mM}$ for SDS and $0.8 \mathrm{mM}$ for CTAB). Further increase of AL, SDS and CTAB concentrations above these optimal values not only represents a waste of dispersants but also induces undesired effects. This is consistent with other studies reporting the aqueous dispersion of CNTs using various surfactants. ${ }^{27,28}$

The well-accepted "unzippering" mechanism can be employed to describe the dispersion of CNTs in aqueous solutions. ${ }^{29}$ Ultrasonication provides a high local shear at the CNT bundle end caused by cavitation. Cavitation occurs during the rarefaction sequence of an applied sonication wave, which causes the formation of solvent cavities or vacuum bubbles when the solution pressure drops below ambient. ${ }^{30}$ These expanding gas pockets collapse due to escalating stresses in their walls, releasing large amounts of energy in the form of high local pressure and temperature, and resulting in liquid jets of up to $280 \mathrm{~m} \mathrm{~s}^{-1}$ velocity. This phenomenon generates gaps between CNT bundles, where dispersant molecules can enter to adsorb onto the newly available CNT surface, hence keeping the connected CNTs partially separated. As the partially individualized CNTs move relative to the bundle due to Brownian motion, the dispersant coating continues to propagate along the CNT length until a complete separation occurs. Suspended single CNTs are considered stable when the interaction between the dispersant and CNT is strong enough to overcome the van der Waals force and $\pi-\pi$ interactions between neighboring CNTs. Depending on the nature of the dispersant, electrostatic repulsion and/or steric hindrance prevent the dispersant-CNT complexes from sticking together. As a result, an equilibrium is reached between single nanotubes and bundles, which varies with concentration. At low concentrations, the dispersant adsorption is limited and does not efficiently counterbalance the van der Waals-induced aggregation of CNTs. However, when the amount of dispersant in the aqueous solution is above a certain value, large micelles form around the CNT bundles and exert an osmotic pressure creating a depletion-induced attraction. ${ }^{31}$ Under similar conditions (i.e. concentration, sonication, temperature...), the variations in dispersion efficiency between different surfactants is closely related to their interaction with CNTs, which depends on the length of their alkyl chain, the presence of benzene rings, and the type of head group. ${ }^{32}$ At the optimal concentrations, the degree of individualized CNTs in water is comparable among the different dispersions, with a slight increase in the order of $\mathrm{AL}>\mathrm{CTAB}>$ SDS, as indicated by their respective absorbance values. While hydrogen bonding can form between hydroxyl groups on AL and polar groups on the CNT surfaces, the adsorption of AL is mostly attributed to the presence of a large number of condensed aromatic structures promoting $\pi-\pi$ interactions with CNTs. ${ }^{26,33}$ The dispersion of CNTs is known to be positively correlated with the number of aromatic rings in the surfactants. Without aromatic units, the uptake of SDS and CTAB on CNTs, which involves relatively weaker hydrophobic interactions, is expected to be lower than that of AL.

\section{Influence of sonication type and energy}

The onset and intensity of cavitation, which is influenced by various factors including the viscosity of the solution and sonication energy, plays a critical role in the dispersion dynamics of CNTs in solution. ${ }^{34}$ Fig. 2 describes the evolution of absorbance for the different aqueous CNT suspensions as
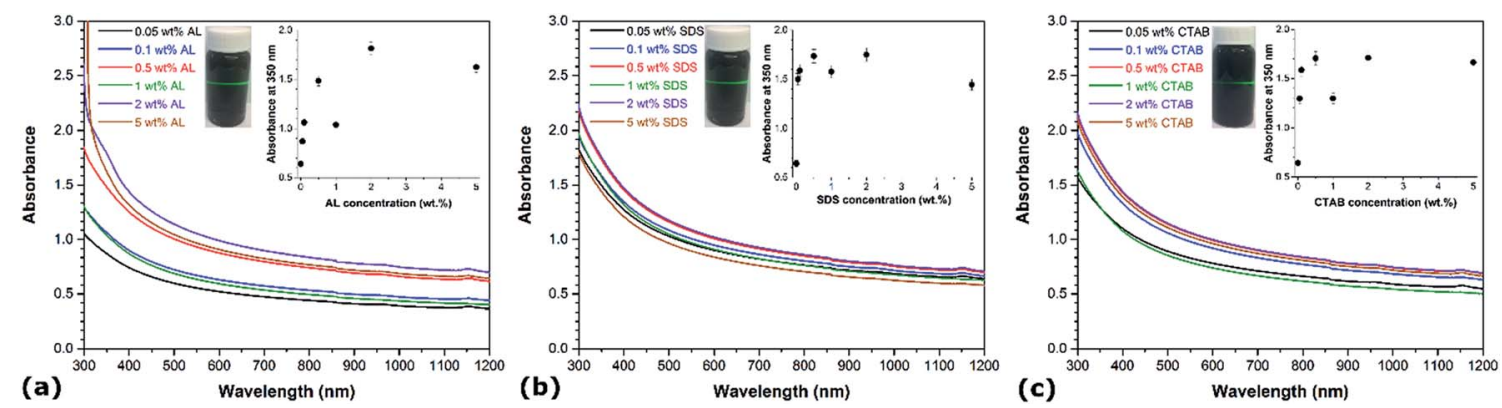

Fig. 1 UV-vis spectra of aqueous CNT suspensions (1 wt\%) using different dispersant concentrations of (a) AL, (b) SDS, and (c) CTAB. Graphs of absorbance at $350 \mathrm{~nm}$ as a function of dispersant concentration and representative photographs of the CNT dispersions are shown in insets. 

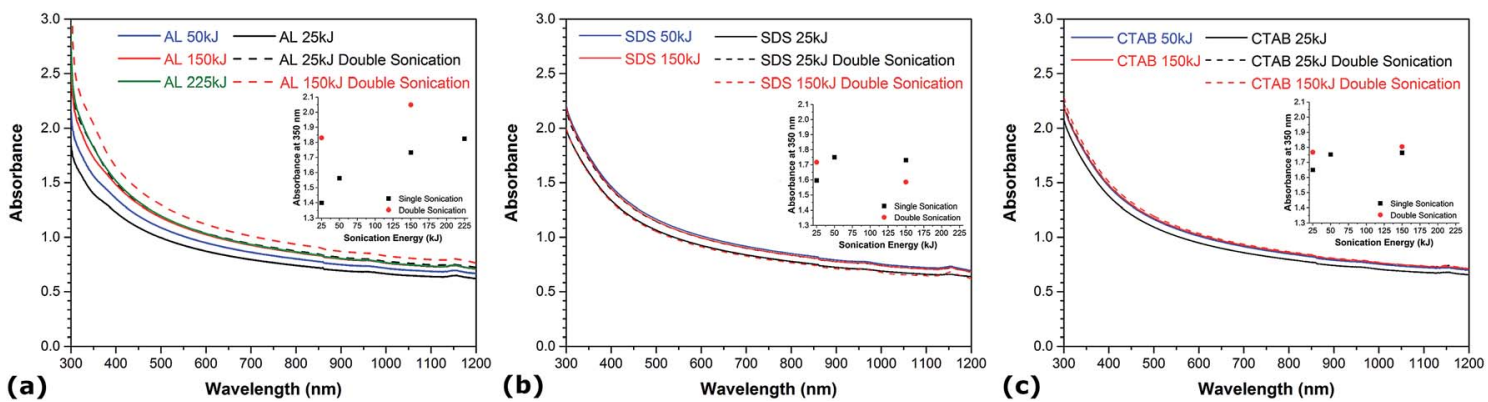

Fig. 2 UV-vis spectra of different aqueous CNT dispersions as a function of the energy supplied to the solution: (a) AL, (b) SDS, and (c) CTAB. Straight lines correspond to probe sonication experiments, while dashed lines represents the double sonication system. Graphs of the absorbance at $350 \mathrm{~nm}$ as a function of sonication type and energy are shown in insets.

a function of the total energy supplied to the solution. In all experiments, the sonication amplitude is kept constant at $28 \%$ and the amount of sonication energy is varied by adjusting the processing time. Comparing the UV-vis spectra of all dispersions obtained at different sonication energy levels, it can be observed that the absorbance increases with sonication energy and reaches a plateau after a certain amount of supplied energy, which corresponds to the maximum achievable degree of CNT individualization in the aqueous solutions. This indicates that there is a minimum energy required to maximally disperse a given amount of CNTs in water, which is consistent with previous work reporting the effect of sonication conditions on the dispersion of CNTs in various solutions. ${ }^{19,33}$ Noteworthy, the value of this minimum sonication energy is higher in the case of $\mathrm{AL}$ (i.e. $\sim 150 \mathrm{~kJ}$ ) than for SDS and CTAB (i.e. $\sim 50 \mathrm{~kJ}$ ), and yields a slightly higher maximum achievable degree of CNT individualization in water. Prolonged acoustic irradiation beyond this threshold is not only energy-consuming, but it also increases the likelihood of damaging the material. Higher sonication energy is known to induce damage and possibly cut the CNTs, which can have negative effects in several applications where CNTs with high aspect ratio are desired. The energy delivered to the solution is related to both sonication duration and amplitude, and it has recently been found that sonication time exhibits a much more pronounced effect on CNT shortening, while variations in the structural integrity of CNTs are limited only to a minor extent with increased amplitude. ${ }^{35}$ Prompted by this, a double acoustic irradiation system combining both bath and probe sonication is developed and investigated. Optical absorption spectra of the double-sonicated solutions (dashed lines) and the probe-sonicated specimens (straight lines) are compared in Fig. 2. At low sonication energy (i.e. $25 \mathrm{~kJ}$ ), a dramatic increase in absorbance is observed in the UV-vis region when the double sonication method is employed independently of the nature of the dispersant. The absorbance values obtained with double sonication at low energy match and even exceed those using traditional probe sonication at higher energy levels. This demonstrates that double acoustic irradiation improves the CNT dispersion process by drastically reducing the processing time while maintaining a very high degree of CNT individualization. The actual power used in the single and double sonication systems was estimated by measuring the temperature rise in an insulated bath in each case. Very little power imparted difference was observed between the use of the double-sonication system and the conventional probe sonication method. Therefore, resonance effects from the different sonication sources are suggested to induce synergies contributing to the enhanced dispersion of CNTs. At higher energy levels (i.e. $150 \mathrm{~kJ}$ ), however, the effect of double sonication is contrasted and depends on the dispersant nature. In the case of SDS and CTAB, the utilization of double sonication at high energy levels yields quantities of single CNTs lower than and comparable to the maximum degree of CNT individualization achieved by typical probe sonication, respectively. For AL, the absorbance obtained by double acoustic irradiation at high energy is significantly increased in the whole UV-vis region with values exceeding the maximum achievable absorbance using traditional probe sonication. This is particularly interesting because this new maximum achievable degree of CNT individualization (i.e. estimated at $500 \mathrm{~nm}$ ) for AL is more than 9 and $12 \%$ higher than that of CTAB and SDS, respectively. Such augmentation beyond the maximum degree of CNT individualization achieved by typical probe sonication can be related to the propensity of lignin to associate into larger macromolecular complexes, which can provide greater repulsive forces between CNTs due to higher spatial volume and more steric hindrance. Acoustic irradiation is known to increase the degree of polymerization of lignin, ${ }^{36}$ hence sonicationinduced polymerization of lignin can enhance the dispersive efficiency of CNTs in water by increasing the spatial volume of lignin molecules adsorbed onto CNTs over the course of the double sonication process. A recent report also showed that higher molecular weight lignin leads to better CNT dispersion than lower molecular weight lignin fractions. ${ }^{33}$ Therefore, the combination of AL with double acoustic irradiation provides an innovative way to both enhance dispersion quality and reduce processing time.

\section{Influence of CNT functionalization and diameter}

The influence of the CNT diameter and surface chemistry on the aqueous dispersion of CNTs are also examined. Fig. 3 shows the UV-vis spectra of the different suspensions prepared with the materials listed in Table 1. In each case, the smaller CNTs (i.e. 

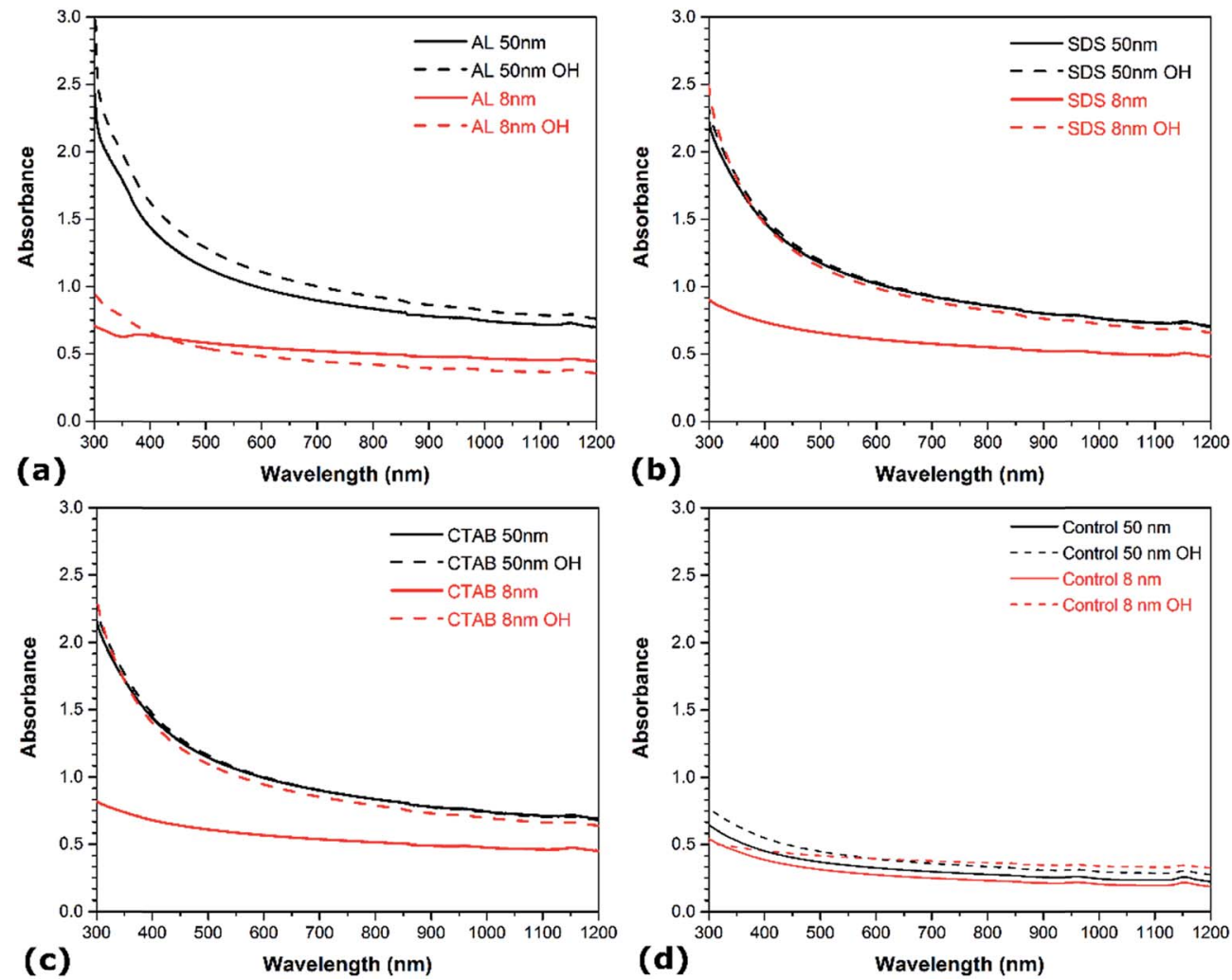

Fig. 3 UV-vis spectra of different aqueous CNT dispersions as a function of CNT diameter and functionalization: (a) AL, (b) SDS, (c) CTAB, and (d) no surfactant.

$8 \mathrm{~nm}$ in diameter) exhibit lower absorbance in the UV-vis region than the larger CNTs (i.e. $50 \mathrm{~nm}$ in diameter). In addition, the aggregates of undispersed $8 \mathrm{~nm}$ CNTs are larger than the aggregates of undispersed $50 \mathrm{~nm}$ CNTs (not shown). The surface contact between nanotubes being larger in the case of CNTs with smaller diameter due to higher surface area and aspect ratio, leads to stronger van der Waals attraction, hence hindering the dispersive efficiency. ${ }^{38,39}$ Surface functionalization also shows a strong influence on the aqueous dispersion of CNTs. In the cases of no surfactant (Fig. 3a), SDS (Fig. 3c), and CTAB (Fig. 3d), the presence of hydroxyl groups increases the absorbance in the UV-vis region. Noteworthy, the absorbance of the $8 \mathrm{~nm}$ functionalized CNTs is in the same range as that of the pristine CNTs with diameter of $50 \mathrm{~nm}$. This indicates that surface-oxidized CNTs are easier to disperse than their nonfunctionalized counterparts under similar conditions. The presence of oxygen functional groups intercalated between the bundles generates stronger steric repulsion between CNTs and facilitates their debundling. This is consistent with recent molecular dynamics simulations showing that oxygen functional groups promote the confinement of polar solvent molecules between graphitic nanomaterials, resulting in higher solvent-induced repulsion for oxidized CNTs, which thus are less prone to aggregate. ${ }^{37}$ Interestingly, the magnitude of the improvement depends on the CNT diameter - surface functionalization exhibits a much more pronounced effect on the dispersion quality of CNTs with larger diameter. In the case of
AL suspensions, however, the influence of surface functionalization is contrasted. The presence of oxygen groups enhances the degree of individualization for the $50 \mathrm{~nm}$ CNTs, but it reduces dispersion quality for CNTs with smaller diameters (i.e. $8 \mathrm{~nm}$ ). The increase in absorbance with surface functionalization for the larger CNTs is also sensibly lower than in the case of SDS and CTAB. These observations reveal that the diameter has a more prominent effect on the CNT suspensions when AL is used as dispersant, while surface oxidation is more influential in SDS and CTAB solutions. This reflects the differences in how these molecules adsorb onto the surface of CNTs. The adsorption of SDS and CTAB surfactants on CNTs occur through electrostatic interactions, which is sensitive to surface oxidation. On the other hand, $\pi-\pi$ interactions are responsible for the adsorption of AL on CNTs, ${ }^{33}$ and larger diameter CNTs exhibit lower surface curvature which leads to stronger affinity to aromatic molecules due to higher overlap between $\pi$ electrons.

\section{Influence of dispersant mixtures}

While most prior work about the aqueous suspension of CNTs have mainly focused on single surfactant solution, the use of mixtures of dispersants can be very effective. For instance, binary mixtures of SDS and CTAB have recently been found to induce synergistic effects improving surfactant packing on surfaces and modifications of repulsive/attractive forces..$^{27,40}$ Here, binary and ternary mixtures of different mixing ratios of 
AL/SDS, AL/CTAB, and AL/SDS/CTAB are studied. Based on the results described above (Fig. 1), all solutions are prepared using a total surfactant to CNT mass ratio of $2: 1$. The optical absorption spectra of the binary and ternary mixtures are presented in Fig. 4a, b and c, respectively. In the case of both binary mixtures (i.e. $\mathrm{AL} / \mathrm{SDS}$ and $\mathrm{AL} / \mathrm{CTAB}$ ), the $\mathrm{AL}$ : surfactant ratio of $90: 10$ achieves higher absorbance than the single dispersant solutions under similar conditions. Comparing the absorbance values of both $90: 10$ binary mixtures, it can be seen that the CNTs dispersed in the AL/SDS solution reach a higher degree of individualization. However, the use of surfactant mixtures does not necessarily guarantee an enhanced dispersing ability, as revealed by both binary mixtures at the $\mathrm{AL}$ : surfactant ratio of $80: 20$, which achieved the lowest absorbance among the different dispersant mixtures with values even below that of single surfactant solutions. Based on this observation, the ratio of $\mathrm{AL}$ is kept at a constant level of 90 in the ternary mixtures. Fig. 4c shows the optical absorption spectra of the different ternary mixtures. While the $90: 1: 9 \mathrm{AL}$ : SDS : CTAB mixture exhibits absorbance values below that of single surfactant solutions, the other ternary mixtures achieved the highest absorbance among all CNT dispersions under similar conditions.

The variations in absorbance due to the addition of CTAB and/or SDS to AL solutions suggests that interactions between these molecules largely contribute to improve the dispersion quality of CNTs in mixed dispersant solutions. The hydrophobic tails of SDS and CTAB molecules can bind to hydrophobic surfaces of lignin, with the head groups that remain in the aqueous phase, providing stabilization by electrostatic repulsion. ${ }^{41}$ This would limit the AL particle size and facilitate the access to adsorption sites located inside the small sonicationgenerated gaps between CNT bundles. The synergistic effects observed in the mixtures with $\mathrm{CTAB}$ can also arise from the electrostatic attractions between surfactants of opposite charge, which can improve dispersant packing onto the surface of CNTs. $^{27}$ However, higher concentrations of SDS or CTAB have been reported to induce the agglomeration of lignin into larger clusters, ${ }^{\mathbf{4 1}}$ which would result in the opposite effect on the adsorption of AL onto CNTs. Reduced surfactant adsorption onto the surface of CNTs ultimately translates to lower dispersion ability. It is also worth noting that the difference of absorbance between pure AL solutions and cationic-rich mixtures (i.e. CTAB ratio $>9$ ) is lower than that between pure $\mathrm{AL}$ and anionic-rich mixtures (i.e. SDS ratio > 9). This can be attributed to the fact that $\mathrm{AL}$ and $\mathrm{CTAB}$ have opposite charges. Therefore, when both molecules adsorb onto CNTs, the charge neutralization leads to a reduction in the electrostatic repulsions between isolated CNTs.

Representative TEM images of different CNTs suspensions depicted in Fig. 5 illustrate the role of AL in dispersing CNTs. The wet TEM specimens were prepared under the same conditions and various types of surfactants were utilized. In the absence of dispersant (Fig. 5a), CNTs are entangled and closely packed with one another, even after being exposed to $150 \mathrm{~kJ}$ of sonication energy. When AL is introduced in the suspension under the same conditions, the degree of CNT individualization significantly increases, as shown in Fig. 5b. Interestingly, it can be observed that some CNTs are partially separated and remain connected at one end, which further exemplifies the abovedescribed "unzippering" mechanism. In the case of dispersant mixtures (Fig. 5c), all CNTs are completely individualized, hence demonstrating the superior dispersion efficiency of surfactant mixtures. It is also worth noting that multiple CNTs have been damaged by high energy sonication, as demonstrated by the magnified view in the inset and by the large number of short CNTs $(<5 \mu \mathrm{m})$.

\section{Colloidal stability of different CNT dispersions in water}

The settling velocity of the aqueous CNT dispersions with pure, binary and ternary surfactants is investigated. While pure CNT suspensions without any dispersant settle down after only few hours, no sedimentation is observed by visual examination of the solutions by the naked eye when surfactants are employed. The dispersion state of the different suspensions as a function of time is characterized by both UV-vis spectroscopy and optical microscopy over a 34 day period, and results shown in Fig. 6 serve as indicators of colloidal stability. Although the absorbance at $500 \mathrm{~nm}$ of all specimens drops in this time period, the most dramatic decreases are observed in the pure SDS and CTAB dispersions with variations up to $21 \%$, which is more than twice the variations of the AL-containing samples (i.e. $<10 \%)$. Moreover, except for the ternary dispersant mixture
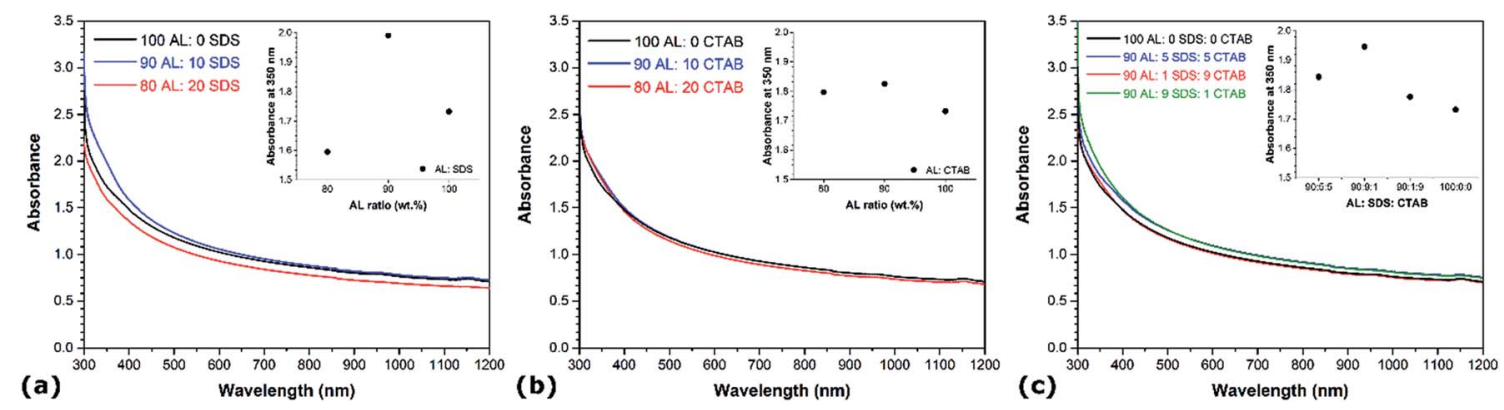

Fig. 4 UV-vis spectra of aqueous CNT suspensions using different binary and ternary dispersant mixtures of (a) AL/SDS, (b) AL/CTAB, and (c) AL/ $\mathrm{SDS} / \mathrm{CTAB}$. The absorption spectra of the CNT dispersion using pure AL (2 wt\%) is added in each figure for comparison purposes. Graphs of the absorbance at $350 \mathrm{~nm}$ as a function of the surfactant composition are shown in insets. 

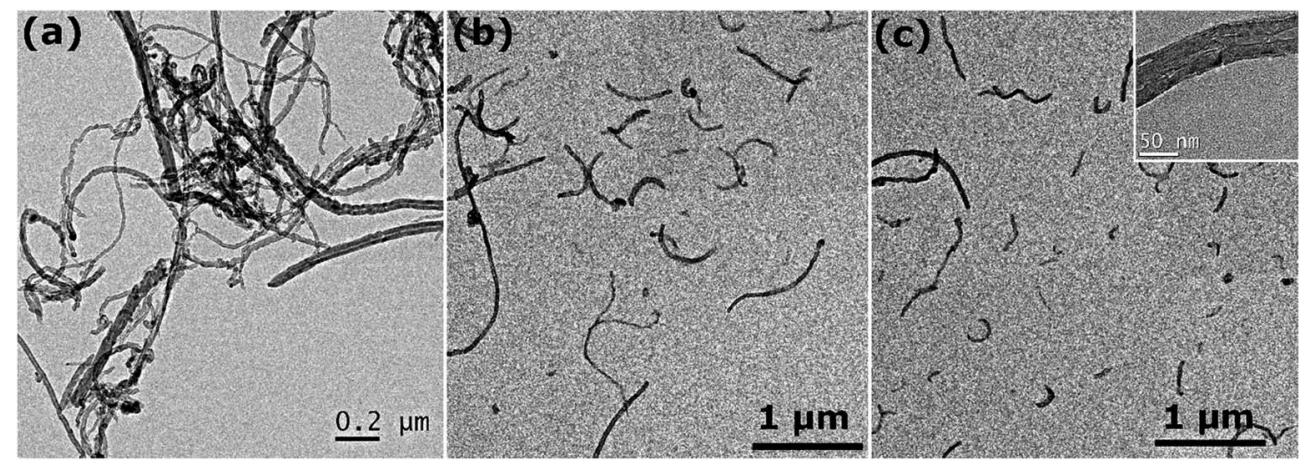

Fig. 5 Representative TEM images of aqueous dispersions of $50 \mathrm{~nm}$ diameter CNTs (a) without any surfactant, (b) with AL, and (c) with a ternary mixture of AL/SDS/CTAB $(90: 9: 1)$.

where the absorbance gradually decreased over time, all other AL-containing specimens exhibit relatively stable absorbance values in the 34 day time period. These results are consistent with light microscope analysis, as depicted in Fig. 6a, b and d. The aggregates observed in the dispersant mixtures (i.e. both binary and ternary solutions) are all smaller than $40 \mu \mathrm{m}$, while an increasing number of aggregates larger than $50 \mu \mathrm{m}$ is found over time in the pure surfactant solutions. The slower settling process of AL-containing suspensions can be attributed to the higher degree of CNT individualization in the solutions, but also the higher viscosity of AL compared to SDS and CTAB. To further study colloidal stability of AL-CNT solutions, the suspensions are dried in air at $50{ }^{\circ} \mathrm{C}$ and re-dispersed in DI water in concentrations ranging from $0.5 \mathrm{wt} \%$ to $2 \mathrm{wt} \%$. The dried CNT powder spontaneously re-disperse itself in water without the need for stirring or sonicating. The resulting suspensions remain stable over several weeks, and centrifugation at $5000 \mathrm{rpm}$ for $30 \mathrm{~min}$ does not induce the precipitation of CNTs. The spontaneous re-dispersion of the dried CNTs in water and their stability at high concentrations are indicative of steric stabilization. Hence, these results demonstrate that the use of AL, either alone or in mixtures with other dispersants, not only achieve a higher degree of CNT individualization in water, but also exhibit enhanced colloidal stability for several weeks compared to traditional petrochemical surfactants.

After processing, the removal of dispersant is usually required in various applications as most surfactants, such as SDS or CTAB, can have detrimental effects on multiple characteristics of the final material. The addition of lignin, however, is known to improve the thermo-mechanical properties of various types of cements, concretes, and polymer composites. ${ }^{42}$ Lignin can act as an adhesion promoter for the formation of highly enhanced interfaces between individualized CNTs and a polymeric matrix. Should the removal of AL be desired for a specific application, it can be efficiently achieved by a simple thermal treatment, as demonstrated in Fig. 7. When the dried AL-CNT powder is annealed in air at $300{ }^{\circ} \mathrm{C}$ for one hour, it does not spontaneously re-dispersed in DI water and settles down almost instantly after stirring. The Tyndall effect is no longer visible in the re-dispersed suspension after annealing, which means that
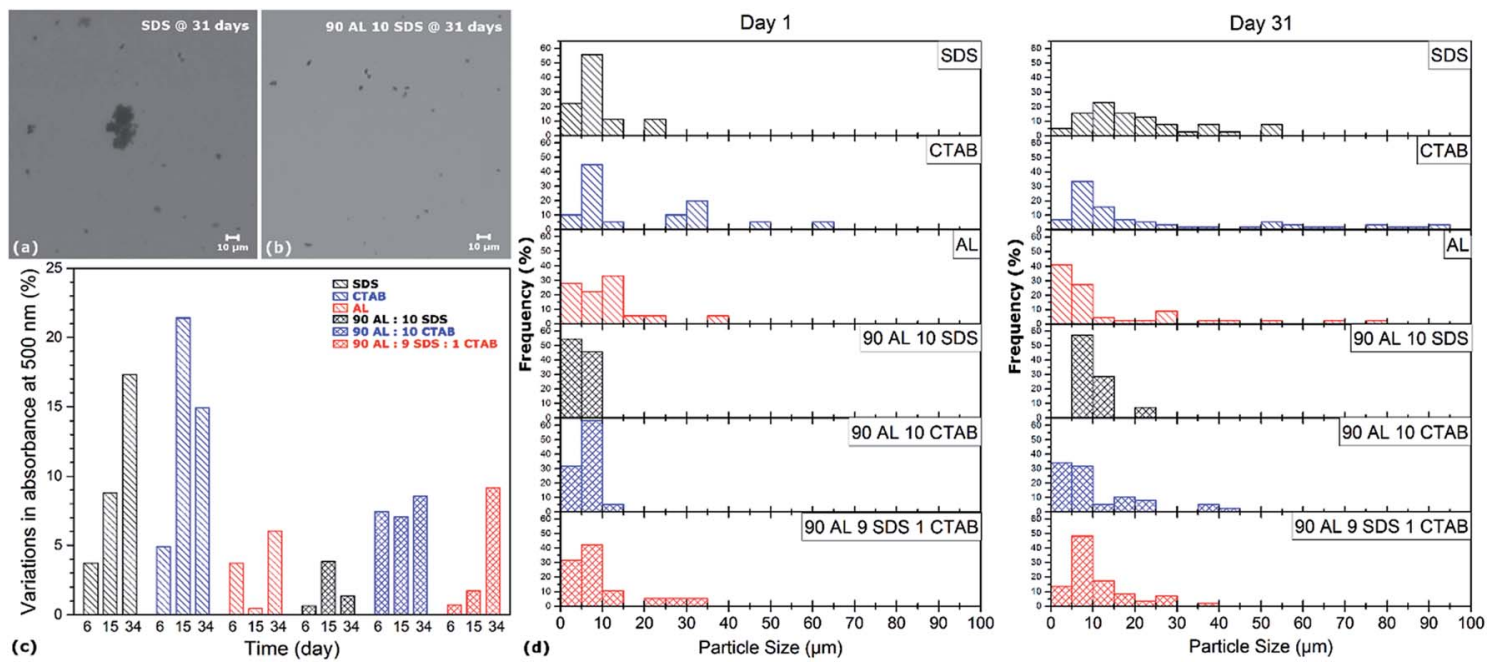

Fig. 6 Representative optical micrographs of aqueous CNT dispersions using (a) SDS and (b) AL/SDS after 1 month sedimentation. (c) Colloidal stability of CNTs suspended in water using different pure, binary, and ternary mixtures of dispersants. (d) Particle size distributions of the different aqueous CNT dispersions after 1 day and 1 month sedimentation. 


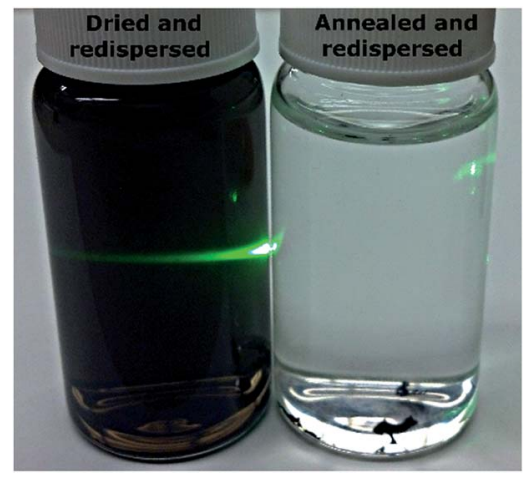

Fig. 7 Vials containing re-dispersed CNTs in water after drying (left) and after annealing at $300{ }^{\circ} \mathrm{C}$ in air for one hour (right). Note that in the right image CNTs coagulate at the bottom and the Tyndall effect is not visible, demonstrating the absence of colloidal particles in water.

colloidal particles are not present in the solution, indicating that AL has been fully removed during thermal treatment.

\section{Conclusion}

The dispersing ability of lignin to prepare aqueous suspensions of CNTs is investigated under different conditions and compared to that of typical petroleum-based surfactants. It is demonstrated from UV-vis spectroscopy and optical microscopy that lignin is effective in dispersing and stabilizing aqueous solutions containing CNTs when compared with other anionic and cationic surfactants. The CNT suspensions remain stable for several weeks with no or very little agglomeration due to both electrostatic and steric stabilization of CNTs by lignin. Results are described based on the well-established "unzippering" mechanism, and show that there is an optimum dispersant : CNT ratio below or above which the quality of CNT suspension deteriorates. Both CNT diameter and surface oxidation are found to play an important role in the dispersion quality. The influence of sonication energy is also studied, and an innovative acoustic irradiation system combining both bath and probe sonication is proposed to improve dispersion efficiency and minimize sonication-induced damages. Although mixed surfactant solutions do not always guarantee an enhanced dispersing ability, synergistic effects are observed in both binary and ternary mixtures with significant improvements in the degree of individualization of CNTs. With this knowledge, the prospects of using lignin containing dispersant mixtures and double sonication systems are expected to increase over the coming years through further research. It is also worth noting that while the removal of surfactant after processing is usually required in various applications as most surfactants can have detrimental effects on the final properties of the material, the addition of lignin can improve the properties of cement, concrete, and polymer composites, such as thermal stability and mechanical strength. ${ }^{42}$ Hence, among the increasing number of natural polymers and biomolecules being used as surfactants or functionalizing agents, lignin represents a major low-cost and renewable candidate for the aqueous dispersion of CNTs. We expect that these findings will guide future optimization of CNT dispersion in aqueous and organic solutions using renewable dispersants. This work also provides relevant data to better assess the environmental fate of aqueous suspensions of CNTs, which are among the most utilized nanomaterials to date.

\section{Acknowledgements}

This work is supported by the USDA National Institute of Food and Agriculture, McIntire Stennis project \#1009515. The authors acknowledge Alissara Holmes for undergraduate research support on this project.

\section{References}

1 P. C. Ma, N. A. Siddiqui, G. Marom and J. K. Kim, Composites, Part A, 2010, 41, 1345-1367.

2 F. Inam, A. Heaton, P. Brown, T. Peijs and M. J. Reece, Ceram. Int., 2014, 40, 511-516.

3 R. H. Schmidt, I. A. Kinloch, A. N. Burgess and A. H. Windle, Langmuir, 2007, 23, 5707-5712.

4 A. Thess, R. Lee, P. Nikolaev, H. Dai, P. Petit, J. Robert, C. Xu, Y. H. Lee, S. G. Kim, A. G. Rinzler, D. T. Colbert, G. E. Scuseria, D. Tománek, J. E. Fischer and R. E. Smalley, Science, 1996, 273, 483-487.

5 J. P. Salvetat, G. A. D. Briggs, J. M. Bonard, R. R. Bacsa, A. J. Kulik, T. Stöckli, N. A. Burnham and L. Forró, Phys. Rev. Lett., 1999, 82, 944-947.

6 W. Li, A. B. Dichiara and J. Bai, Compos. Sci. Technol., 2013, 74, 221-227.

7 M. S. de Luna, L. Pellegrino, M. Daghetta, C. V. Mazzocchia, D. Acierno and G. Filippone, Compos. Sci. Technol., 2013, 85, 17-22.

8 A. B. Dichiara, J. Yuan, S. Yao, A. Sylvestre, L. Zimmer and J. Bai, J. Mater. Chem. A, 2014, 2, 7980-7987.

9 O. V. Kharissova, B. I. Kharisov, V. M. Jiménez-Pérez, B. M. Flores and U. O. Méndez, RSC Adv., 2013, 3, 2264822682.

10 S. Kim, Y. I. Lee, D. H. Kim, K. J. Lee, B. S. Kim, M. Hussain and Y. H. Choa, Carbon, 2013, 51, 346-354.

11 M. Sheikholeslam, M. Pritzker and P. Chen, Carbon, 2014, 71, 284-293.

12 Z. Song, J. Dai, S. Zhao, Y. Zhou, F. Su, J. Cui and Y. Yan, RSC Adv., 2014, 4, 2327-2338.

13 M. Zheng, A. Jagota, E. D. Semke, B. A. Diner, R. S. Mclean, S. R. Lustig, R. E. Richardson and N. G. Tassy, Nat. Mater., 2003, 2, 338-342.

14 R. M. F. Fernandes, B. Abreu, B. Claro, M. Buzaglo, O. Regev, I. Furo and E. F. Marques, Langmuir, 2015, 31, 10955-10965.

15 Y. Bai, D. Lin, F. Wu, Z. Wang and B. Xing, Chemosphere, 2010, 79, 362-367.

16 J. Rausch, R. C. Zhuang and E. Mader, Composites, Part A, 2010, 41, 1038-1046.

17 W. H. Duan, Q. Wang and F. Collins, Chem. Sci., 2011, 2, 1407-1413.

18 A. Payen, Comptes Rendus, 1838, 7, 1052-1056. 
19 O. Rochez, G. Zorzini, J. Amadou, M. Claes and A. Richel, J. Mater. Sci., 2013, 48, 4962-4964.

20 P. Estellé, S. Halelfadl and T. Maré, Int. Commun. Heat Mass Transfer, 2014, 57, 8-12.

21 A. Pénicaud, F. Dragin, G. Pécastaings, H. Maoshaui and E. Anglaret, Carbon, 2014, 67, 360-367.

22 P. Alafogianni, K. Dassios, S. Farmaki, S. K. Antiohos, T. E. Matikas and N. M. Barkoula, Colloids Surf., A, 2016, 495, 118-124.

23 H. Kataura, Y. Kumazawa, Y. Maniwa, I. Umezu, S. Suzuki, Y. Ohtsuka and Y. Achiba, Synth. Met., 1999, 103, 2555-2558.

24 J. S. Lauret, C. Voisin, G. Cassabois, C. Delalande, P. Roussignol, O. Jost and L. Capes, Phys. Rev. Lett., 2003, 90, 057404.

25 S. Attal, R. Thiruvengadathan and O. Regev, Anal. Chem., 2006, 78, 8098-8104.

26 W. Liu, R. Zhou, D. Zhou, G. Ding, J. M. Soah, C. Y. Yue and X. Lu, Carbon, 2015, 83, 188-197.

27 B. Sohrabi, N. Poorgholami-Bejarpasi and N. Nayeri, J. Phys. Chem. B, 2014, 118, 3094-3103.

28 R. Rastogi, R. Kaushal, S. K. Tripathi, A. L. Sharma, I. Kaur and L. M. Bharadwaj, J. Colloid Interface Sci., 2008, 328, 421-428.

29 M. S. Strano, V. C. Moore, M. K. Miller, M. J. Allen, E. H. Haroz, C. Kittrell, R. H. Hauge and R. E. Smalley, J. Nanosci. Nanotechnol., 2003, 3, 81-86.

30 B. Vijayendran, D. Mangaraj and D. Ensminger, Ultrasonics: Data, Equations and Their Practical Uses, ed. F. B. Stulen and D. Ensminger, CRC Press, Boca Raton, 2008, ch. 8, pp. 323364.
31 B. Vigolo, A. Pénicaud, C. Coulon, C. Sauder, R. Pailler, C. Journet, P. Bernier and P. Poulin, Science, 2000, 290, 1331-1334.

32 L. Vaisman, H. D. Wagner and G. Marom, Adv. Colloid Interface Sci., 2006, 128-130, 37-46.

33 N. Y. Teng, I. Dallmeyer and J. F. Kadla, Ind. Eng. Chem. Res., 2013, 52, 6311-6317.

34 J. Yu, N. Grossiord, C. E. Koning and J. Loos, Carbon, 2007, 45, 618-623.

35 R. Fuge, M. Liebscher, C. Schröfl, S. Oswald, A. Leonhardt, B. Buchner and V. Mechtcherine, Diamond Relat. Mater., 2016, 66, 126-134.

36 T. Wells, M. Kosa and A. J. Ragauskas, Ultrason. Sonochem., 2013, 20, 1463-1469.

37 N. S. Azar and M. Pourfath, J. Phys. Chem. C, 2016, 120, 16804-16814.

38 J. G. Duque, A. N. G. Parra-Vasquez, N. Behabtu, M. J. Green, A. L. Higginbotham, B. K. Price, A. D. Leonard, H. K. Schmidt, B. Lounis, J. M. Tour, S. K. Doorn, L. Cognet and M. Pasquali, ACS Nano, 2010, 4, 3063-3072.

39 M. Bystrzejewski, A. Huczko, H. Lange, T. Gemming, B. Büchner and M. H. Rümmeli, J. Colloid Interface Sci., 2010, 345, 138-142.

40 Y. Bai, J. Gao, C. Wang, R. Zhang and W. Ma, J. Nanosci. Nanotechnol., 2016, 16, 2239-2245.

41 H. Stewart, M. Golding, L. Matia-Merino, R. Archer and C. Davies, Food Res. Int., 2014, 66, 93-99.

42 V. K. Thakur, M. K. Thakur, P. Raghavan and M. R. Kessler, ACS Sustainable Chem. Eng., 2014, 2, 1072-1092. 